Maria da Conceição Nascimento Costa ${ }^{1}$

\title{
Mortalidade infantil e condições de vida: a reprodução das desigualdades sociais em saúde na década de 90
}

\author{
Infant mortality and living conditions: \\ the reproduction of social inequalities \\ in health during the 1990s
}

Paula de Almeida Azi 1

Jairnilson Silva Paim 1

Lígia Maria Vieira da Silva 1

\footnotetext{
1 Instituto de Saúde Coletiva, Universidade Federal da Bahia. Rua Padre Feijó 29, 4 o andar, Salvador, $B A$ 40110-170, Brasil. mcncosta@ufba.br
}

\begin{abstract}
An ecological study was conducted to determine the infant mortality trend from 1991 to 1997 and to analyze its relationship to living conditions in Salvador, Bahia State, Brazil. Inequality patterns in infant death were analyzed by spatial distribution and a compound socioeconomic index. The data showed a decline in the infant mortality rate, with neonatal deaths and perinatal causes playing a growing role. Despite this overall trend, the infant mortality rate increased in 1992, and it was only in 1997 that it returned to the 1991 level. This fact was interpreted as related to worsening living conditions during that period. Spatial distribution highlights the persistence of health inequalities; education was the variable with the most significant correlation rate. When distributed according to the living conditions index (LCI), both the infant mortality rate and proportional infant mortality showed a linear increase from the intermediate stratum (20.4\%o) to the lowest (29.3\%o) and from the highest stratum (5.3\%) to the lowest (13.3\%), respectively. The authors conclude that despite the reduction in the total infant mortality rate, the persistence of social inequalities and a social process that hinders improvement of living conditions are responsible for the inequalities observed in infant mortality.
\end{abstract}

Key words Infant Mortality; Social Conditions; Spatial Analysis

Resumo O estudo descreve a evolução da mortalidade infantil em Salvador, de 1991 a 1997, $e$ analisa as desigualdades nestas mortes considerando-se sua distribuição espacial e um índice de condições de vida. Houve um declínio da mortalidade, passando as mortes neonatais e as causas perinatais a ter maior importância. Em 1992, o coeficiente de mortalidade infantil (CMI) aumentou 75\%, e só em 1997 voltou aos patamares de 1991, fenômeno que os autores atribuíram à deterioração das condições de vida. A análise da distribuição espacial aponta para a manutenção das desigualdades sociais, sendo a educação a variável de maior correlação. A mortalidade infantil proporcional (MIP) e o CMI mostraram um crescimento linear a partir do estrato de elevada (5,3\%) para o de muito baixa (13,3\%), e do estrato de intermediária para o de muito baixa condições de vida (de 20,4\%o para 29,3\%o), respectivamente. Conclui-se que, apesar da redução dos níveis da mortalidade infantil, são mantidas as desigualdades sociais em saúde, e que os processos sociais que comprometem as condições de vida continuam desempenhando um relevante papel na sua determinação.

Palavras-chave Mortalidade Infantil; Condições Sociais; Análise Espacial 


\section{Introdução}

A estreita relação que a mortalidade infantil apresenta com os fatores sociais e econômicos é reconhecida há muito tempo e tem sido evidenciada em diversos estudos latino-americanos (Behm, 1980; Monteiro, 1982; Paim et al., 1987; Yunes, 1983). Em virtude da grande vulnerabilidade que as crianças com menos de um ano de idade apresentam em face das alterações ocorridas no ambiente social e econômico e das intervenções de saúde (Murray, 1988), a mortalidade nessa faixa etária é considerada como um indicador tanto da situação de saúde, quanto das condições de vida de uma população (Grant, 1992). Entretanto, estudos realizados em países em desenvolvimento têm demonstrado que essa vinculação deixou de ser tão evidente, visto que, apesar da crise econômica mundial observada a partir dos anos 80, não ocorreu uma reversão da tendência decrescente que esta mortalidade vinha exibindo (Ageitos et al., 1991).

Na América Latina, uma das conseqüências dessa crise foi o agravamento das desigualdades sociais. Todavia, em vários países verificouse a manutenção e mesmo uma intensificação da queda que vinha sendo registrada nas taxas de mortalidade infantil (Silva \& Duran, 1990). Esse panorama contribuiu para que as questões relativas às desigualdades em saúde passassem a ser privilegiadas na demonstração empírica dos diferenciais sócio-econômicos do processo saúde-doença e, por conseguinte, na identificação de grupos populacionais submetidos a riscos mais elevados (Breilh, 1990). Algumas organizações internacionais de saúde passaram a orientar os investigadores no sentido de dirigir seus esforços para a elaboração de novos métodos e técnicas que possibilitassem discriminar melhor a situação de saúde segundo as condições de vida (OPS, 1992; WHO, 1991).

Diante das dificuldades para operacionalizar o conceito de classe social, alguns autores têm se inclinado para o emprego de indicadores compostos por diferentes variáveis sócioeconômicas que permitem uma aproximação das condições materiais de existência de grupos humanos de uma sociedade (Castellanos, 1990). Outra abordagem empregada na apreensão dos processos envolvidos na determinação da doença na população tem sido aquela na qual as relações sociais também são entendidas como determinantes do padrão de ocupação do espaço de uma cidade. Assim, os indicadores tradicionais de saúde são estimados para áreas geográficas com menor nível de agregação, tendo como referência, ainda que nem sempre explicitada, a consideração de que o padrão espacial da cidade é definido pelas relações sociais decorrentes do modo de produção econômica (Santos, 1980), e que a evolução do espaço é concomitantemente uma condição e uma conseqüência da evolução de uma sociedade global (Santos, 1979).

Paim (1997) imputa às condições de vida de cada classe social o papel de mediação dos determinantes estruturais da saúde. Para operacionalizar esse conceito, utiliza indicadores sociais e econômicos selecionados, considerando que uma abordagem a partir dos distintos espaços da cidade, ocupados por diferentes grupos populacionais, permite uma aproximação da realidade, apesar da sua complexidade.

Embora seja bastante antigo o emprego da distribuição espacial para descrever a ocorrência da doença, em geral, essa abordagem restringia-se à comparação de diferenças internacionais e regionais, em que fatores ambientais/ climáticos eram apresentados como principais determinantes das desigualdades encontradas. Mais recentemente, essa estratégia vem sendo uma das mais utilizadas para analisar a influência de fatores demográficos, sócio-econômicos e de atenção à saúde na determinação e explicação da variação da mortalidade infantil (Gonçalo-Perez \& Herrera-Leon, 1990; Lardelli et al., 1993; Zurriaga-Llorens et al., 1990).

No Brasil, esse tipo de enfoque revelou uma grande heterogeneidade na distribuição espacial dos óbitos de menores de um ano no Rio Grande do Sul (Victora et al., 1994), em Porto Alegre (Guimarães \& Fischmann, 1986) e em São Paulo (Monteiro et. al., 1980; Yunes, 1983).

Também em Salvador, Bahia, em 1980, foi demonstrada uma acentuada desigualdade na ocorrência das mortes infantis quando distribuídas nas diferentes zonas de informação desse município (Paim et al., 1987), que se manteve entre os anos 1980-1988, apesar de os níveis desta mortalidade terem decrescido (Paim \& Costa, 1993).

Considerando a complexidade e o dinamismo dos processos que envolvem a mortalidade infantil, faz-se necessário o seu contínuo acompanhamento, de modo que se possa dispor de informações que permitam a análise da situação de saúde no nível local e a adoção de medidas de controle pertinentes. Assim, o presente trabalho tem como objetivos descrever a evolução da mortalidade infantil em Salvador entre os anos de 1991 e 1997 e analisar a relação existente entre a distribuição espacial dessa mortalidade e as condições de vida da população do município em 1991 e 1994. 


\section{Metodologia}

O estudo, do tipo ecológico e de base populacional, teve como menor unidade de análise as 75 zonas de informação (ZI) de Salvador, que correspondem a áreas da cidade definidas pela Companhia de Desenvolvimento da Região Metropolitana (CONDER) com base em critérios sócio-econômicos, de infra-estrutura físicoadministrativa e de planejamento, compatibilizados com os setores censitários da Fundação Instituto Brasileiro de Geografia e Estatística (IBGE).

Foram utilizados os dados do Sistema de Informação de Mortalidade (SIM) contidos no CD-ROM produzido pelo Ministério da Saúde (MS, 1998). Todos os óbitos de maiores de um ano ou de idade ignorada que tiveram como causa básica as afecções originadas no período perinatal, foram, no presente estudo, considerados como sendo de menores de um ano, ocorridos no período neonatal.

Para análise da distribuição espacial, empregaram-se as Declarações de Óbito (DO) de residentes em Salvador, Bahia, dos anos de 1991 e 1994, arquivadas no Centro de Informações de Saúde da Secretaria de Saúde do Estado da Bahia (CIS/SESAB). Além do endereço habitual dos falecidos, para os menores de um ano, foram levantados dados como sexo, data do nascimento e de óbito, idade ao falecer e a causa básica já selecionada e codificada no CIS/SESAB. O endereço da residência habitual do falecido registrado em cada DO foi codificado e classificado por ZI. A descrição detalhada dos procedimentos desta classificação encontra-se publicada (Paim et al., 1987), diferindo apenas quanto às zonas 15 e 25, que foram agrupadas em virtude de mudanças nos setores censitários efetuadas pelo IBGE para o Censo de 1991 (IBGE, 1991), e às respectivas adaptações realizadas pela CONDER.

O estudo da relação entre a distribuição espacial da mortalidade infantil e alguns fatores sócio-econômicos foi feito apenas para 1991, porque somente para este ano dispunha-se de indicadores desta natureza para as ZI. Com este propósito, buscou-se uma aproximação das condições sócio-econômicas e de atenção à saúde das ZI por meio da distribuição das seguintes variáveis: proporção de indivíduos de 10 a 14 anos alfabetizados (educação); proporção de chefes de famílias em domicílios permanentes com rendimento médio mensal igual ou menor que dois salários mínimos (renda); proporção de domicílios com canalização interna ligados à rede geral de abastecimento de água (saneamento) e percentagem de casas em “aglomerado subnormal” em relação ao total de domicílios (favela). Todas essas variáveis são provenientes do IBGE, bem como do número de postos e centros de saúde e de leitos obstétricos por mil habitantes, fornecidos pelo CIS/ SESAB.

O acompanhamento da evolução da mortalidade foi realizado por intermédio da comparação da magnitude dos indicadores globais, de grupos e causas específicas e dos componentes neonatal e pós-neonatal nos anos investigados, e, também, com base na observação da variação da mortalidade infantil, tomando-se como referência os quartis constituídos por ZI que em 1980 foram classificados como de baixa, intermediária, elevada e muito elevada mortalidade infantil proporcional (MIP) (Paim et al., 1987). Após o cálculo do valor deste indicador para o ano de 1991 nesses mesmos quartis, estimou-se o percentual de variação da mortalidade no período, tendo como parâmetros os valores encontrados em estudo anterior (Paim \& Costa, 1993) para os anos de 1980 e 1988.

A análise dos dados foi ainda efetuada valendo-se de uma agregação das ZI, que teve como eixo o índice de condições de vida (ICV) (Paim, 1987). Este é um indicador composto, cujo escore final resultou da pontuação por ZI nas variáveis renda, educação, saneamento e favela, já definidas anteriormente, e mais a $\mathrm{RM} / \mathrm{Q}$, que corresponde à razão entre o número médio de moradores por domicílio (moradores) e o número médio de cômodos servindo de dormitório (quarto) nas respectivas ZI.

A ordenação dos escores de cada ZI permitiu que estas fossem agrupadas em quatro estratos (quartis) constituídos por áreas relativamente homogêneas de condições de vida: elevada - escores de 18 a 60; intermediária - escores variando de 61 a 107; baixa - escores de 108 a 146; muito baixa - escores de 147 a 219 . Optou-se pela estratificação em quartis porque, do ponto de vista estatístico, tomando-se a mediana como referência, mantém-se o equilíbrio da divisão na medida em que cada um dos grupos fica composto por $25 \%$ das observações. Para este trabalho, foram distribuídos nos respectivos estratos a população de menores de um ano, o número de óbitos totais e de menores de um ano, estimando-se a seguir os indicadores de mortalidade infantil estudados.

A análise das mortes infantis com base na variação da sua magnitude, considerando os quartis da MIP em 1980 e da sua relação com o ICV, foi inviabilizada para 1994 porque 460 $(35,2 \%)$ DO de menores de um ano, daquele ano, não apresentaram o registro do endereço 
de residência do falecido $(32,8 \%)$ ou este estava incompleto $(2,4 \%)$.

A mortalidade infantil proporcional (MIP), o coeficiente de mortalidade infantil (CMI) e de seus componentes e a proporção de óbitos de menores de um ano por grupos de causas e causas específicas foram os indicadores de mortalidade empregados na análise dos dados. O estudo das mortes ocorridas em 1996 e 1997, por capítulos da Classificação Internacional de Doenças (CID), foi feita em separado dos demais anos porque a 10a Revisão da CID foi adotada a partir de 1996, e modificações nesta poderiam interferir na tendência da mortalidade por causas, o que na realidade não corresponderia a uma alteração do padrão epidemiológico (Grassi \& Laurenti, 1998).

Utilizou-se como denominador para o cálculo do CMI a população de menores de um ano fornecida pelo IBGE para os anos de 1991 e 1996; para os demais, foram feitas estimativas pelo método geométrico, valendo-se dos dados dos censos demográficos de 1980, 1991 e 1996. Fez-se essa opção em virtude de o Sistema de Informações de Nascidos Vivos (SINASC) só ter disponibilizado o número de nascidos vivos a partir de 1994, pela inexistência dessa informação por ZI, e também pela pouca confiabilidade das estimativas disponíveis sobre esse número para o Município de Salvador.

O processamento dos dados e a estimativa dos intervalos de confiança dos CMI dos estratos de condições de vida foram efetuados com o programa Epi-Info 6.0 (WHO, 1994). Para verificar a existência de associação entre as variáveis independentes selecionadas e os indicadores de mortalidade (MIP e CMI) das ZI, calculou-se o coeficiente de correlação de Spear- man, utilizando-se o programa SPSS for Windows 9.0 (SPSS Incorporation, 1998).

Para o cálculo do coeficiente de correlação, foram excluídas algumas ZI cuja população com menos de um ano de idade era igual ou inferior a cem, a fim de evitar a instabilidade dos indicadores em virtude dos pequenos números envolvidos. Enquadraram-se nessa situação as ZI 3 (Ondina), 9 (Vitória/Campo Grande), 14 (Canela), 23 (Frederico Pontes/Petrobrás), 35 (Avenida Heitor Dias/Acesso Norte), 45 (CAB Centro Administrativo da Bahia), 56 (Aeroporto/Praias do Flamengo/Stella Mares), 72 (Limite com a USIBA), 74 (Ilhas: Capeta, Bom Jesus dos Frades) e 76 (Ilha de Maré).

\section{Resultados}

Observa-se na Tabela 1 que, em Salvador, no ano de 1991, ocorreram 1.111 óbitos de menores de um ano, o que correspondeu ao CMI de 29,2 por mil menores de um ano e MIP de $10,2 \%$. A proporção de óbitos neonatais (PON) foi praticamente a mesma da pós-neonatal (POPN), de $49,1 \%$ e $50,1 \%$, respectivamente. Em 1992, houve um aumento da mortalidade infantil, expressa por todos os seus indicadores, inclusive no número absoluto de óbitos e em seus dois componentes. Em termos percentuais, essa elevação foi de $75 \%$ no CMI, 55,9\% na MIP, $82 \%$ na PON e de $75 \%$ na POPN, quando comparados aos valores apresentados no ano anterior. Em que pese à redução dos níveis da mortalidade a partir de 1993, a sua magnitude manteve-se até 1996, sempre superior à observada em 1991, e somente em 1997, quando o CMI foi de $27,9 \%$ e a MIP de $8,2 \%$, atingiu patamares in-

Tabela 1

Mortalidade infantil, neonatal e pós-neonatal. Salvador, 1991-1997.

\begin{tabular}{|c|c|c|c|c|c|c|c|c|c|}
\hline \multirow{2}{*}{ Ano } & \multicolumn{3}{|c|}{ Mortalidade infantil } & \multicolumn{3}{|c|}{ Mortalidade neonatal } & \multicolumn{3}{|c|}{ Mortalidade pós-neonatal } \\
\hline & Óbitos & Coef. 1 & MIP & Óbitos & Coef. 1 & $\% 2$ & Óbitos & Coef. 1 & $\% 2$ \\
\hline 1991 & 1.111 & 29,2 & 10,2 & 545 & 14,3 & 49,1 & 566 & 14,9 & 50,1 \\
\hline 1992 & 1.922 & 51,1 & 15,9 & 982 & 26,1 & 51,1 & 940 & 25,0 & 48,9 \\
\hline 1993 & 1.776 & 47,8 & 14,1 & 963 & 25,9 & 54,2 & 813 & 21,9 & 45,8 \\
\hline 1994 & 1.588 & 43,2 & 12,2 & 914 & 24,9 & 57,6 & 674 & 18,3 & 42,4 \\
\hline 1995 & 1.264 & 34,8 & 10,8 & 830 & 22,8 & 65,7 & 434 & 12,0 & 34,3 \\
\hline 1996 & 1.113 & 31,0 & 8,6 & 690 & 19,2 & 62,0 & 423 & 11,8 & 38,0 \\
\hline 1997 & 994 & 27,9 & 8,2 & 671 & 18,8 & 67,5 & 323 & 9,1 & 32,5 \\
\hline
\end{tabular}

1 Coeficiente por 1.000 menores de um ano.

2 Proporção em relação ao total de óbitos de menores de um ano.

Fonte: Ministério da Saúde/CD-ROM. 
feriores ao do início desta série. Nota-se, ainda, que, apesar deste comportamento observado na mortalidade infantil entre 1992 e 1996, foi justamente a partir de 1992 que passou a haver um predomínio da mortalidade neonatal, de forma sempre crescente (a exceção do ano de 1996), de modo que, no ano de 1997, esse componente da mortalidade infantil já representava $67,5 \%$ dos óbitos de menores de um ano.

Quatro grupos de causas foram responsáveis por mais de $85 \%$ dos óbitos de menores de um ano, entre 1991 e 1995, por ordem de importância: afecções originadas no período perinatal, doenças infecciosas e parasitárias (DIP), doenças do aparelho respiratório (DAR) e anomalias congênitas. Em 1996 e 1997, de acordo com a CID 10, esses quatro grupos de causas de óbito contribuíram respectivamente com $88,7 \%$ e $90 \%$ de todas as mortes infantis; as anomalias congênitas ocuparam a terceira posição em 1996 e a segunda no ano seguinte, ocasião em que as DIP e as DAR passaram, respectivamente, para os terceiro e quarto lugares. Chama ainda a atenção o comportamento do número de óbitos por causas externas, que foi de apenas cinco em 1991, elevou-se para 17 e 23 nos dois anos posteriores e, a partir de então, até 1997, variou entre 10 e 12 .

Quando se considera a participação de algumas causas específicas de óbitos por capítulo da CID, verifica-se que, em 1991, dos 247 óbitos de menores de um ano por DIP, 189 $(76,5 \%)$ corresponderam às infecções intestinais mal definidas e 49 (19,8\%), às septicemias. Em 1994 e 1997, esses valores foram respectivamente de $224(77,8 \%)$ e $51(60,0 \%)$ para as infecções intestinais mal definidas e de $52(18,1 \%)$ e $23(27,1 \%)$ para as septicemias. Entre os óbitos por DAR, $123(80,9 \%), 158(78,6 \%)$ e $53(67,1 \%)$ foram causados por infecções respiratórias agudas, principalmente broncopneumonias, em cada um dos três anos; quanto aos óbitos por afecções originadas no período perinatal, observou-se que, em 1991, 98 (21,\%), 94 (20,2\%) e $44(9,4 \%)$ deveram-se a, respectivamente, infecções específicas do recém-nascido, hipóxia intra-uterina e asfixia ao nascer, bem como aos transtornos relacionados a gestação de curta duração e peso baixo ao nascer de tipo não especificado. Em 1994, esses valores foram de 109 (13,7\%), 99 (12,4\%) e 91 (1,4\%), enquanto, para 1997, foram de 114 (19\%), 67 (11,2\%) e 29 (4,8\%), para cada uma das referidas causas.

No ano de 1991, não ocorreram óbitos de menores de um ano entre os residentes nas ZI 9 (Campo Grande/Vitória), 13 (Horto Florestal) e 21 (Itaigara). Nas demais, os valores eram elevados, com CMI atingindo até $60 \%$ de menores de um ano, como nas ZI 68 (Valéria) (66,0\%) e 47 (Bonfim/Ribeira/M. Serrat) (60,3\%). Em cerca de um terço das sessenta ZI com população de menores de um ano superior a cem, o valor desse indicador superou o da média da cidade $(28,8 \%)$, e, em $40 \%$ destas ZI, a MIP ultrapassou $10 \%$, chegando inclusive a representar $25 \%$ dos óbitos de menores de um ano na ZI 52 (Mata Escura), e 20\% na 71 (Coutos).

Observou-se uma associação positiva, estatisticamente significante $(p=0,01)$, entre a MIP e renda baixa $(r=0,55)$, proporção de casas em favelas $(r=0,52)$ e número de centros e postos de saúde $(r=0,32)$. Observou-se ainda uma significante $(\mathrm{p}=0,01)$ associação negativa com educação $(r=-0,72)$, proporção de casas ligadas à rede de abastecimento de água $(\mathrm{r}=-0,40)$ e número de leitos obstétricos $(r=-0,21)$, porém, com esta última, não houve significância estatística ( $p=0,09)$. Já o CMI só se mostrou correlacionado com o número de leitos obstétricos $(\mathrm{r}=0,30 ; \mathrm{p}=0,01)$ e com o número de centros e postos de saúde $(r=0,36 ; p=0,003)$.

A análise da evolução da MIP segundo quartis, no período compreendido entre 1980 e 1991, revelou menor declínio no quartil de baixa MIP (-47,5\%) e maior no de intermediária MIP $(68,2 \%)$, a magnitude desta não diferindo em muito da redução apresentada pelos quartis de elevada e muito elevada MIP. No período compreendido entre 1988 e 1991, a menor redução $(31,7 \%)$ também ocorreu no quartil de baixa MIP, e a seguir no de muito elevada MIP $(39,2 \%)$; por outro lado, o maior declínio (-50,7\%) foi apresentado pelo quartil de elevada MIP (Tabela 2).

Nos agregados constituídos com base no ICV, a proporção de óbitos neonatais foi de $55 \%$ no estrato de elevada condição de vida, reduzindo-se para menos de $50 \%$ nos demais e alcançando apenas $38,2 \%$ naquele de pior condição de vida. Por sua vez, a MIP mostrou-se

Tabela 2

Mortalidade infantil proporcional (MIP) e variação percentual em períodos de tempo especificados, nos quartis de zonas de informação de baixa, intermediária, elevada e muito elevada MIP em 1980. Salvador, 1980, 1988, 1991.

\begin{tabular}{lrrrrrr}
\hline $\begin{array}{l}\text { Quartis da MIP } \\
\text { em 1980 }\end{array}$ & \multicolumn{3}{c}{ MIP } & \multicolumn{3}{c}{ Variação (\%) } \\
& 19801 & 19881 & 1991 & $1980-881$ & $1988-91$ & $1980-91$ \\
\hline Baixa & 11,8 & 9,0 & 6,2 & $-23,7$ & $-31,7$ & $-47,5$ \\
Intermediária & 21,7 & 12,9 & 6,9 & $-44,7$ & $-46,5$ & $-68,2$ \\
Elevada & 31,4 & 21,5 & 10,6 & $-31,5$ & $-50,7$ & $-66,5$ \\
Muito elevada & 42,5 & 22,7 & 13,8 & $-46,6$ & $-39,2$ & $-67,5$ \\
\hline
\end{tabular}

1 Paim \& Costa, 1993. 
sempre crescente do estrato de condição elevada $(5,3 \%)$ para o de muito baixa $(13,3 \%)$; porém, ao se tratar do risco de morte antes de completar um ano de idade, um crescimento linear uniforme só se deu a partir do estrato de intermediária (20,4\%) para o de muito baixa condição de vida (29,3\%), já que no de ICV elevado encontrou-se o maior coeficiente de mortalidade infantil $(31,3 \%)$ entre os quatro estratos (Tabela 3; Figura 1).

Com esse tipo de agregação, observou-se ainda que as afecções perinatais, as DIP, as DAR e as anomalias congênitas, nesta ordem, constituíram-se nos quatro principais grupos de causas de mortes de menores de um ano em todos os estratos, variando apenas quanto a sua magnitude, a qual, na maioria das vezes, foi maior naqueles agregados onde eram piores as condições de vida. Particularizando cada um desses grupos de causas, pode-se verificar que as afecções originadas no período perinatal representa- ram $44,1 \%$ dos óbitos infantis no estrato de condição elevada, sendo inferior a $37 \%$ nos demais. O maior risco de morrer por essas causas $(13,8 \%)$ também foi apresentado pelo estrato de elevada, e o menor, no estrato de condições de vida intermediárias $(7,5 \%)$. Praticamente, não houve diferença nos valores do coeficiente de mortalidade pelas infecções específicas do recémnascido, hipóxia ao nascer, anóxia intra-uterina e transtornos relativos a gestação de curta duração e baixo peso ao nascer de tipo não especificado, entre os estratos de elevada (5,9\%) e muito baixa $(5,1 \%)$, como também entre os de intermediária $(4,2 \%)$ e baixa $(4,8 \%)$ condição de vida.

No que diz respeito às DIP, o menor e o maior riscos foram encontrados respectivamente nos estratos de intermediária $(3,8 \%)$ e de muito baixa $(7,7 \%)$ condição de vida, e os estratos de elevada e de baixa condição apresentaram para estas causas CMI do mesmo valor $(5,6 \%)$. Do total desses óbitos em cada estrato, $95,0 \% \mathrm{e}$

Alguns indicadores demográficos e de mortalidade em estratos de zonas de informação de residência, constituídos com base nos quartis dos índices de condições de vida (ICV). Salvador, 1991.

\begin{tabular}{|c|c|c|c|c|}
\hline & \multicolumn{4}{|c|}{ Estratos de condição de vida } \\
\hline & Elevada & Intermediária & Baixa & Muito baixa \\
\hline \multicolumn{5}{|l|}{ Indicadores } \\
\hline População $<1$ ano & 3.551 & 7.313 & 13.680 & 13.460 \\
\hline Óbitos de $<1$ ano & 111 & 149 & 354 & 395 \\
\hline Óbitos neonatais (\%) & 55,0 & 49,0 & 43,8 & 38,2 \\
\hline Mortalidade infantil proporcional & 5,3 & 7,1 & 10,9 & 13,3 \\
\hline Coeficiente de mortalidade infantil & 31,31 & 20,42 & 25,93 & 29,34 \\
\hline \multicolumn{5}{|l|}{ Mortalidade $<1$ ano por } \\
\hline \multicolumn{5}{|c|}{ Afecções originadas no período perinatal } \\
\hline Coeficiente & 13,8 & 7,5 & 9,4 & 7,7 \\
\hline$\%$ & 41,4 & 41,2 & 41,4 & 26,9 \\
\hline \multicolumn{5}{|l|}{ Doenças infecciosas e parasitárias } \\
\hline Coeficiente & 5,6 & 3,8 & 5,6 & 7,4 \\
\hline$\%$ & 20,2 & 18,8 & 21,8 & 27,8 \\
\hline \multicolumn{5}{|l|}{ Doenças do aparelho respiratório } \\
\hline Coeficiente & 3,4 & 3,3 & 4,5 & 4,7 \\
\hline$\%$ & 12,1 & 16,1 & 17,5 & 17,0 \\
\hline \multicolumn{5}{|l|}{ Anomalias congênitas } \\
\hline Coeficiente & 3,4 & 2,3 & 2,4 & 2,3 \\
\hline$\%$ & 12,1 & 11,4 & 9,3 & 8,4 \\
\hline
\end{tabular}

Nota: Foram excluídos 87 óbitos de menores de um ano,

por terem o endereço de residência ignorado ou não registrado na DO.

1 IC $95 \% 25,9 \quad 37,4$

2 IC $95 \% 17,3 \quad 23,8$

3 IC $95 \% 23,3 \quad 28,6$

4 IC95\% 26,6 32,3 
$97,4 \%$ foram causados por infecções intestinais mal definidas e septicemias nos estratos de elevada e baixa condição de vida, e de $100 \%$ nos de condição intermediária e muito baixa.

Para os óbitos decorrentes de doenças do aparelho respiratório, os riscos variaram pouco, sendo respectivamente de $3,4 \%$ e de $3,3 \%$ nos agregados de elevada e intermediária condição de vida, e de $4,5 \%$ e $4,7 \%$ nos de baixa e muito baixa condição. A proporção de óbitos por infec- ções respiratórias agudas (IRA) em cada um dos estratos de ICV variou de 79,1\% do total de óbitos infantis por DAR do estrato de elevada a $69,8 \%$ no de muito baixa condição de vida, e o coeficiente de mortalidade cresceu de $2,5 \%$ para 3,3\% do estrato de baixa para o de muito elevada condição vida. Quanto às mortes por anomalias congênitas, o maior risco $(3,4 \%)$ ocorreu no estrato de elevada condição de vida (Tabela 3; Figura 2). Observou-se, ainda, que somente

\section{Figura 1}

Coeficiente de mortalidade infantil (por 1.000 menores de um ano) e mortalidade infantil proporcional (\%), segundo estratos de condições de vida. Salvador, 1991.

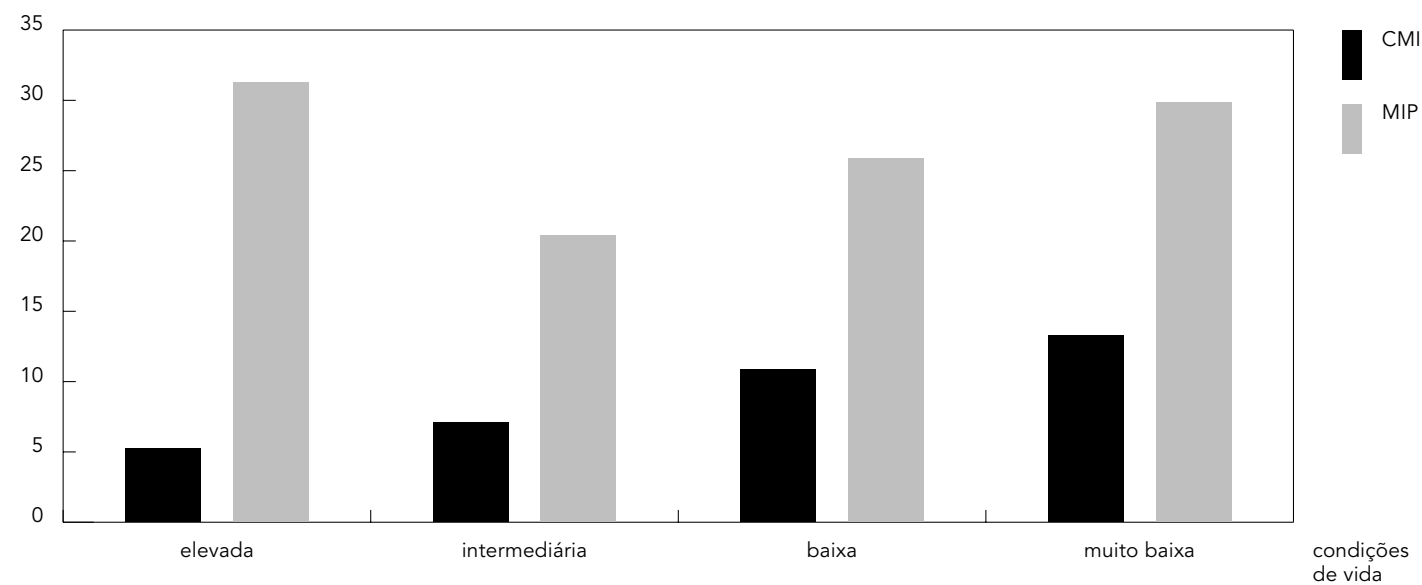

Figura 2

Coeficiente de mortalidade infantil de (por 1.000 menores de um ano) pelos quatro principais grupos de causas, segundo estratos de condições de vida. Salvador, 1991.

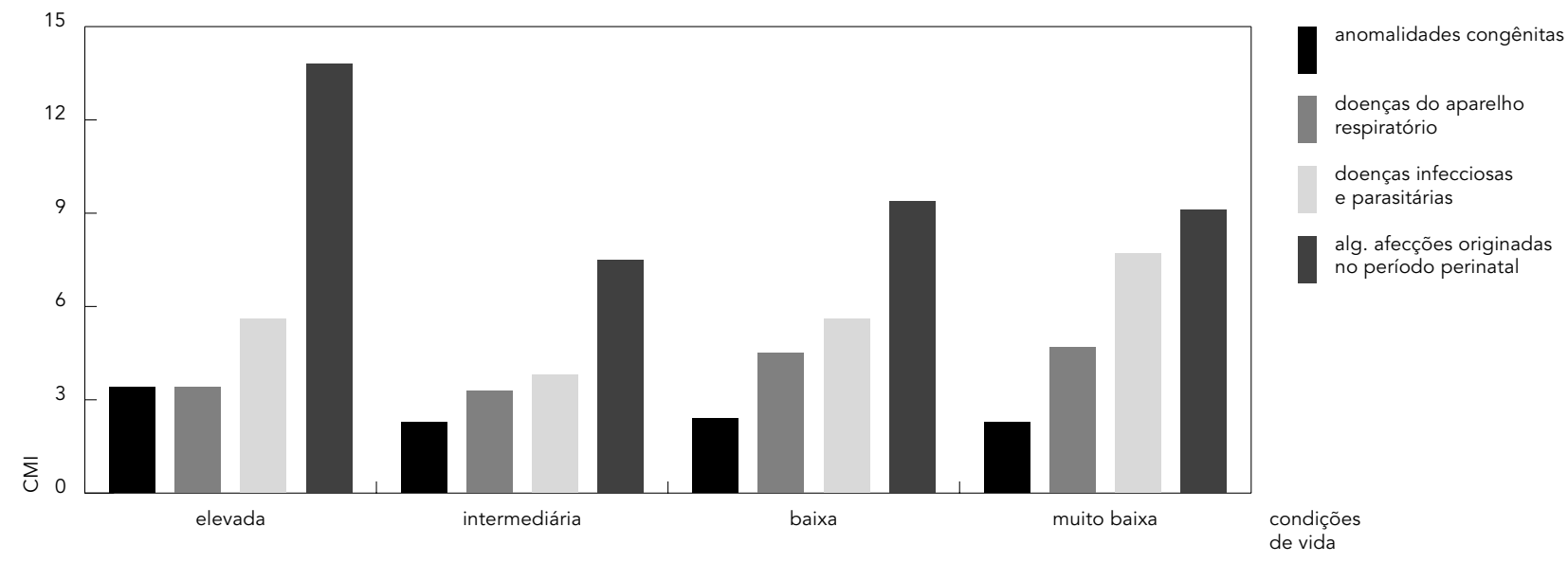


no estrato de elevado ICV não houve registro de óbitos de menores de um ano classificados como decorrentes de causas mal definidas. Essa distribuição dos indicadores em cada estrato manteve-se mesmo quando foram definidos estratos pelo cálculo de tercil e quintil dos escores do ICV.

\section{Discussão}

Os resultados deste estudo confirmam o declínio que vinha sendo observado na mortalidade infantil em Salvador (SESAB, 1995; Lima \& Costa, 1999). Todavia, mostram também que essa queda não foi contínua e apresentou uma variabilidade muito grande. Entre 1991 e 1992, houve uma elevação da ordem de $75 \%$ no coeficiente de mortalidade infantil e de $55,5 \%$ na participação dos óbitos dos menores de um ano em relação ao total de óbitos de todas as idades. Observou-se, inclusive, que, entre 1992 e 1995, os níveis dessa mortalidade foram similares aos observados nos últimos anos da década de 80 (Lima \& Costa, 1999). É grande a possibilidade de que esse aumento da mortalidade infantil tenha sido real, pois não há indícios de ter havido no período qualquer melhoria no sistema de informação de saúde no Estado da Bahia.

O emprego da população de menores de um ano, em vez do número de nascidos vivos, para o cálculo do coeficiente de mortalidade infantil, por um lado, favorece uma superestimação deste indicador, e, por outro, dificulta a sua comparação com os de outros locais onde esse procedimento não foi adotado. Contudo, considerando que a população de menores de um ano é próxima à de nascidos vivos, e que ainda há algum sub-registro de óbitos de menores de um ano no município em estudo, certamente não se estará incorrendo em um erro muito grande ao se afirmar que o risco de morte de menores de um ano em Salvador ainda pode ser considerado elevado se confrontado com os apresentados em países desenvolvidos (WHO, 1993), e mesmo com os de regiões mais desenvolvidas do Brasil (SES-RS, 1993).

Além da acentuada redução que vem ocorrendo na mortalidade infantil, é clara a modificação do padrão da distribuição dos óbitos por grupos de causas, particularmente a partir de 1981, quando as afecções originadas no período perinatal passaram a predominar, possivelmente em decorrência da redução dos óbitos por doenças infecciosas e parasitárias e por doenças do aparelho respiratório (SESAB, 1991). Tais fatos podem estar refletindo a influência da adoção de algumas medidas de atenção à saúde, como o programa de controle das infec- ções respiratórias agudas, a terapia de reidratação oral e o incentivo à amamentação, entre outros, conforme já assinalados em estudo anterior (Paim \& Costa, 1993). Porém, a redução da mortalidade, sem que haja uma melhoria das condições de vida da população, está sujeita a sofrer uma reversão toda vez que o sistema de saúde não atender adequadamente às necessidades e/ou deteriorarem-se ainda mais as condições de vida. É o que pode ter ocorrido em 1992, pois o país sofreu uma acentuada queda do Produto Interno Bruto (PIB), da ordem de $4 \%$ em 1990 e de $6,87 \%$ entre abril de 1990 e março de 1991 (Anuário Delta Universal, 1992). No Estado da Bahia, a variação do PIB foi de -1,5\% (SEI, 1999), podendo essa recessão ter comprometido as condições sócio-econômicas da população.

No caso em questão, além da ascensão dos níveis da mortalidade infantil quando comparados aos já apresentados em 1990 (Lima \& Costa, 1999) e 1991, observou-se que tanto as causas perinatais, como as doenças infecciosas e parasitárias e do aparelho respiratório, contribuíram para a inversão observada na tendência dessa mortalidade em 1992, o que é sugestivo da instabilidade em que se encontra a situação de saúde infantil e das condições de vida da população.

A constatação de que, além dos óbitos por DIP, a maioria daqueles por DAR tinha origem infecciosa, e de que, entre as causas de mortes perinatais, encontram-se várias outras que poderiam não ter ocorrido, caso houvesse adequada cobertura e qualidade da assistência ao pré-natal, ao parto e ao recém-nascido, permite afirmar que a persistência das mortes por causas evitáveis no nosso meio é uma realidade. Se forem considerados apenas os óbitos por doenças infecciosas intestinais, septicemias e infecções específicas do recém-nascido, hipóxia intra-uterina, asfixia ao nascer e transtornos relacionados a gestação de curta duração e baixo peso ao nascer, verifica-se que o número de óbitos infantis em Salvador ainda poderia ser reduzido em pelo menos mais um terço, mesmo em 1997, quando o nível da mortalidade infantil já era o mais baixo desta década.

Como já observado em anos anteriores (Paim et al., 1987; Paim \& Costa, 1993), houve uma distribuição bastante desigual dos óbitos dos menores de um ano no espaço geográfico do município em 1991. As áreas com ausência ou baixa freqüência dessas mortes estão localizadas mais para o sul e ao leste da cidade e corresponderam às zonas nas quais os indicadores sócio-econômicos mostraram-se mais favoráveis. Outras áreas, detentoras de elevados índices de mortalidade infantil, são ocupadas principalmente por uma população menos favore- 
cida e se situam nas zonas mais centrais, periféricas e do sentido norte da cidade. Em geral, os coeficientes de mortalidade infantil nestas áreas eram iguais ou muito próximos daqueles observados em alguns países pobres da África, como a Argélia e a Namíbia em 1992 (UNICEF, 1994).

Diferentemente do que foi demonstrado em outro estudo da mortalidade infantil nesse mesmo município em 1980 (Paim et al., 1987), a associação da MIP com a renda, em 1991, foi mais fraca do que a encontrada para a educação, apontando para as modificações que podem ocorrer na dinâmica dos fatores que medeiam a mortalidade em conjunturas diversas. É possível que a inexistência ou pouca associação das variáveis independentes estudadas com o coeficiente de mortalidade infantil deva-se a uma maior sensibilidade do coeficiente de correlação e à natureza das unidades de medida envolvidas na construção desses indicadores. A MIP reflete a posição das mortes infantis em relação ao total de mortes, logo apenas os óbitos estão influenciando. Já no coeficiente de mortalidade infantil, cujo valor aproxima-se do risco de morrer antes de completar um ano de idade, além dos óbitos, há também a influência do tamanho e do grau de homogeneidade da população menor de um ano.

A distribuição espacial dos leitos obstétricos também favoreceu as áreas de melhores condições de vida, podendo a alta cobertura $(98,5 \%)$ de partos hospitalares observada no município (Barros et al., 1993) ter contribuído para a fraca associação negativa desses recursos com a MIP. Por outro lado, a contraditória associação positiva com o número de centro e postos de saúde pode ter sido decorrente tanto do fato de estas unidades estarem localizadas nas áreas mais pobres da cidade, que também são as de maior mortalidade infantil, como de uma cobertura insuficiente e/ou baixa utilização e resolutividade dos serviços prestados por essas unidades de saúde. Em apoio parcial a esta hipótese, tem-se a informação de que em um Distrito Sanitário de Salvador, em 1992, a taxa global de utilização de serviços de saúde pela população foi de apenas $12,5 \%$, e que os serviços públicos desse Distrito foram responsáveis por somente $25,9 \%$ do total de atendimentos (Vieira-da-Silva et al., 1995).

O quadro observado no agregado de elevada condição de vida apresenta algumas contradições que merecem ser discutidas, como, por exemplo, a magnitude superior à dos demais estratos do risco da mortalidade infantil global e do risco de morte por DIP igual ao verificado no estrato de baixa condição de vida. Esse perfil, em parte, pode ser resultante apenas de um artefato numérico, hipótese que se fundamen- ta na pequena precisão apresentada pelo CMI neste estrato e que, possivelmente, deve-se ao menor substrato encontrado para atuação do risco da mortalidade infantil (a população menor de um ano era, neste estrato, cerca de duas a quase quatro vezes menor que a dos demais). Além disso, deve ser salientado que todas as crianças menores de um ano, denominador utilizado no cálculo do CMI, encontram-se distribuídas nos respectivos estratos, enquanto no numerador deixou de ser incluído um certo número de óbitos de menores de um ano, por não ter sido possível classificá-los por ZI. Como é maior a probabilidade de que esses óbitos tenham sido de residentes em áreas mais pobres, isto significa que o risco de morte infantil dos estratos de piores condições de vida pode estar apresentando um maior grau de subestimação que aqueles de melhores condições sócio-econômicas. A migração de óbitos (registro de óbitos de recém-nascidos de mães residentes em áreas mais pobres no estrato de elevada condição de vida) e a provável existência de um maior sub-registro de óbitos nos estratos de mais baixas condições de vida, principalmente daquelas crianças consideradas como natimortas, embora tenham sobrevivido por alguns segundos, também são suposições plausíveis.

Além dos aspectos referentes à qualidade e à quantidade da informação, pode-se ainda atribuir esses achados à diferença da qualidade da assistência oferecida pelos serviços de saúde ao parto e aos recém-nascidos de mães pertencentes ao estrato de elevada condição de vida. Parte dos reflexos dessa diferença poderia também se fazer sentir no menor sub-registro, além do melhor diagnóstico da causa do óbito.

Por fim, não se deve deixar de referir mais um fator que pode ter influenciado nos resultados. É o fato de o ICV ter sido construído com base em valores médios de alguns indicadores sócio-econômicos, estando, portanto, sujeito às mesmas restrições que este tipo de medida (médias). Desse modo, a heterogeneidade no interior da própria zona de informação, aliada às diferenças entre cada uma delas que compõem o respectivo estrato, pode estar ajudando a conformar esse quadro, tendo em vista que principalmente o estrato de elevada condição de vida admitiu, na sua composição, a inclusão de zonas de informação como a 29 (Beiru/Cabula), 15/25 (Centro Histórico/Nazaré), 20 (Brotas), 33 (Caixa d'Água) e 38 (Calçada/Mares/ Roma/Boa Viagem), que apresentaram um número de óbitos infantis bastante superior ao das demais ZI componentes deste agregado e/ ou indicadores sócio-econômicos que, isoladamente, eram mais desfavoráveis (Tabela 4). 
Tabela 4

Alguns indicadores demográficos, sócio-econômicos e de atenção à saúde, segundo zonas de informação. Salvador, 1991.

\begin{tabular}{|c|c|c|c|c|c|c|c|c|c|c|c|c|c|c|c|}
\hline Zona de informação & ICV & $\begin{array}{l}\text { Pop. } \\
<1 \text { ano }\end{array}$ & $\begin{array}{l}\text { Óbito } \\
\text { totais }\end{array}$ & $\begin{array}{l}\text { Óbito } \\
<1 \text { ano }\end{array}$ & $\begin{array}{l}\text { Óbito< } \\
\text { 28dias }\end{array}$ & MIP & CMI & CMNN & CMPNN & $\begin{array}{l}\text { Educ. } \\
\text { fund. }\end{array}$ & $\begin{array}{l}\text { Renda } \\
\text { baixa }\end{array}$ & $\begin{array}{c}\text { Centros/ } \\
\text { Postos }\end{array}$ & $\begin{array}{c}\text { L.Obst/ } \\
1.000\end{array}$ & $\begin{array}{l}\text { Saneam. } \\
\text { (2) }\end{array}$ & Favela \\
\hline 1- Barra & 1 & 200 & 133 & 6 & 3 & 4,5 & 30,0 & 15,0 & 15,0 & 97,4 & 10,1 & - & 1,4 & 99,3 & 1,7 \\
\hline 2- Jardim Apipema & 1 & 135 & 58 & 2 & 2 & 3,4 & 14,8 & 14,8 & - & 96,5 & 14,1 & 1 & - & 99,9 & 4,9 \\
\hline 3- Ondina & 1 & 60 & 46 & 4 & 1 & 11,1 & 66,7 & 16,7 & 50,0 & 94,2 & 20,2 & - & - & 99,7 & 0,0 \\
\hline 4/5- Rio Vermelho & $2 / 1$ & 271 & 145 & 7 & 5 & 4,8 & 25,8 & 18,5 & 6,3 & 95,71 & 26,41 & 1 & 1,1 & 99,81 & 2,01 \\
\hline 8- Pituba & 1 & 389 & 221 & 12 & 4 & 5,4 & 30,8 & 10,3 & 20,5 & 96,9 & 9,0 & - & - & 99,9 & 0,1 \\
\hline $\begin{array}{l}\text { 9- Campo Grande/ } \\
\text { Vitória }\end{array}$ & 1 & 61 & 59 & - & - & - & - & - & - & 96,7 & 7,8 & - & - & 93,1 & 0,0 \\
\hline 10- Graça & 1 & 108 & 85 & 1 & 1 & 1,2 & 9,3 & 9,3 & - & 97,5 & 7,7 & - & - & 99,4 & 0,1 \\
\hline 14- Canela & 1 & 31 & 37 & 4 & 1 & 10,8 & 129,0 & 32,2 & 96,8 & 95,0 & 9,4 & - & - & 100,0 & 4,4 \\
\hline $\begin{array}{l}\text { 15/25- Centro } \\
\text { Hist./Nazaré }\end{array}$ & $1 / 1$ & 358 & 360 & 22 & 14 & 6,1 & 61,5 & 39,1 & 22,4 & 94,61 & 26,61 & 1 & 4,6 & 99,91 & 2,71 \\
\hline 20- Brotas & 1 & 493 & 420 & 20 & 14 & 4,8 & 40,6 & 28,4 & 12,2 & 92,3 & 4,3 & 1 & 2,2 & 99,9 & 7,1 \\
\hline $\begin{array}{l}21-\text { Pq. N. S. Luz/ } \\
\text { Itaigara }\end{array}$ & 1 & 299 & 49 & - & - & - & - & - & - & 96,9 & 9,2 & - & - & 99,7 & 1,1 \\
\hline 26- Matatu & 1 & 174 & 117 & 5 & 2 & 4,3 & 28,7 & 11,5 & 17,2 & 95,1 & 22,6 & 1 & - & 99,9 & 0,1 \\
\hline 29- Cabula & 1 & 363 & 166 & 16 & 9 & 9,6 & 44,1 & 24,8 & 19,3 & 97,2 & 24,5 & 3 & 2,9 & 99,9 & 0,4 \\
\hline 33- Caixa D'água & 1 & 459 & 97 & 10 & 5 & 10,3 & 21,8 & 10,9 & 10,9 & 93,7 & 48,9 & 1 & 0,7 & 99,8 & 0,5 \\
\hline 38- Calçada/Mares & 1 & 199 & 152 & 4 & 1 & 2,6 & 20,1 & 5,0 & 15,1 & 92,4 & 44,9 & - & - & 99,8 & 0,4 \\
\hline 46- Piatã/Patamares & 1 & 50 & 23 & 1 & 1 & 4,3 & 20,0 & 20,0 & - & 98,6 & 11,6 & 1 & - & 99,7 & 0,0 \\
\hline 56- P. do Flamengo & 1 & 41 & - & - & - & - & - & - & - & 96,1 & 7,3 & - & - & 73,5 & 0,3 \\
\hline 6- Amaralina & 2 & 502 & 54 & 6 & 4 & 11,1 & 12,0 & 8,0 & 4,0 & 90,2 & 55,0 & - & - & 99,6 & 0,1 \\
\hline $\begin{array}{l}\text { 11/12- Alto das } \\
\text { Pombas }\end{array}$ & $4 / 2$ & 1.185 & 358 & 26 & 13 & 7,3 & 21,9 & 11,0 & 10,9 & 89,91 & 60,81 & 3 & 0,7 & 98,31 & 9,91 \\
\hline 13- Horto Florestal & 2 & 204 & 7 & - & - & - & - & - & - & 94,6 & 30,8 & - & - & 99,8 & 7,3 \\
\hline 16- Garcia & 2 & 188 & 110 & 4 & 1 & 3,6 & 21,3 & 5,3 & 16,0 & 92,7 & 40,5 & 1 & - & 99,7 & 11,5 \\
\hline 17- Barra/Fonte Nova & 2 & 152 & 60 & 5 & 2 & 8,3 & 32,9 & 13,2 & 19,7 & 94,4 & 49,1 & - & - & 96,7 & 0,5 \\
\hline 18- Eng. V. de Brotas & 2 & 553 & 155 & 10 & 6 & 6,5 & 18,1 & 10,8 & 7,3 & 92,5 & 22,4 & - & - & 99,2 & 4,6 \\
\hline 19- Acupe & 2 & 415 & 52 & 5 & 3 & 9,6 & 12,0 & 7,2 & 4,8 & 92,5 & 22,4 & - & - & 99,7 & 6,1 \\
\hline 22- Stiep/Armação & 2 & 410 & 68 & 5 & 2 & 7,4 & 12,2 & 4,9 & 7,3 & 96,6 & 69,3 & - & - & 99,9 & 0,1 \\
\hline 23- Frederico Pontes & 2 & 20 & 16 & 1 & - & 6,3 & 50,0 & - & 50,0 & 87,0 & 57,0 & - & - & 98,5 & 0,0 \\
\hline $\begin{array}{l}\text { 24- Chile/ Baixa } \\
\text { dos Sapateiros }\end{array}$ & 2 & 211 & 91 & 11 & 1 & 12,1 & 52,1 & 4,7 & 47,4 & 89,1 & 57,0 & 1 & - & 99,9 & 0,1 \\
\hline $\begin{array}{l}\text { 28- Luis Anselmo/ } \\
\text { V Laura }\end{array}$ & 2 & 340 & 28 & 1 & 1 & 3,6 & 2,9 & 2,9 & - & 93,0 & 32,1 & - & - & 99,7 & 3,3 \\
\hline 32- Barbalho/Lapinha & 2 & 260 & 153 & 8 & 4 & 5,2 & 30,8 & 15,4 & 15,4 & 90,8 & 45,2 & 1 & - & 99,9 & 5,4 \\
\hline $\begin{array}{l}\text { 34- Quintas/ } \\
\text { Cidade Baixa }\end{array}$ & 2 & 677 & 237 & 10 & 5 & 4,2 & 14,8 & 7,4 & 7,4 & 93,1 & 63,6 & 2 & 1,5 & 99,6 & 2,5 \\
\hline 35- Acesso Norte & 2 & 6 & 24 & - & - & - & - & - & - & 93,4 & 60,4 & - & - & 97,4 & 2,0 \\
\hline 36- $19 \mathrm{BC}$ & 2 & 353 & 37 & 5 & 2 & 13,5 & 14,2 & 5,7 & 8,5 & 91,2 & 38,8 & - & - & 99,5 & 16,1 \\
\hline 39/40- Liberdade & $3 / 2$ & 1.453 & 603 & 44 & 16 & 7,3 & 30,3 & 11,0 & 19,3 & 91,61 & 62,81 & 2 & - & 99,51 & 2,41 \\
\hline 47- Bonfim/Ribeira & 2 & 365 & 243 & 22 & 17 & 9,1 & 60,3 & 46,6 & 13,7 & 95,0 & 35,6 & 1 & 1,9 & 98,4 & 3,3 \\
\hline 50/51- São Caetano & $3 / 2$ & 1.602 & 408 & 36 & 19 & 8,8 & 22,5 & 11,9 & 10,6 & 88,91 & 58,91 & 3 & - & 99,51 & 3,31 \\
\hline 27- Cosme de Farias & 3 & 671 & 200 & 14 & 8 & 7,0 & 20,9 & 11,9 & 9,0 & 87,1 & 68,2 & 1 & - & 99,7 & 0,1 \\
\hline 31- Boca do Rio & 3 & 693 & 215 & 16 & 7 & 7,4 & 23,1 & 10,1 & 13,0 & 88,3 & 52,1 & 1 & - & 99,4 & 13,3 \\
\hline 37- Pituaçu & 3 & 277 & 32 & 4 & - & 12,5 & 14,4 & - & 14,4 & 87,6 & 45,7 & 1 & - & 99,7 & 21,4 \\
\hline 41- IAPI & 3 & 553 & 170 & 15 & 8 & 8,8 & 27,1 & 14,5 & 12,6 & 87,9 & 57,5 & 1 & - & 99,1 & 24,2 \\
\hline 42- Fazenda Grande & 3 & 775 & 264 & 29 & 13 & 11,0 & 37,4 & 16,8 & 20,6 & 88,3 & 63,6 & 1 & - & 99,5 & 5,3 \\
\hline 43- S. G. do Retiro & 3 & 378 & 85 & 8 & 3 & 9,4 & 21,2 & 7,9 & 13,3 & 86,5 & 55,1 & - & - & 99,7 & 28,0 \\
\hline 48- Jardim Cruzeiro & 3 & 924 & 248 & 20 & 11 & 8,1 & 21,6 & 11,9 & 9,7 & 90,8 & 65,2 & 3 & - & 98,3 & 10,8 \\
\hline $\begin{array}{l}\text { 54- Paralela/ } \\
\text { E. V. Aeroporto }\end{array}$ & 3 & 1.150 & 86 & 14 & 5 & 16,3 & 12,2 & 4,3 & 7,9 & 83,3 & 42,6 & 4 & - & 99,4 & 10,4 \\
\hline 58- Campinas & 3 & 514 & 39 & 2 & - & 5,1 & 3,9 & - & 3,9 & 86,8 & 61,6 & - & - & 99,4 & 7,3 \\
\hline
\end{tabular}

(continua) 
Tabela 4 (continuação)

\begin{tabular}{|c|c|c|c|c|c|c|c|c|c|c|c|c|c|c|c|}
\hline Zona de informação & ICV & $\begin{array}{l}\text { Pop. } \\
<1 \text { ano }\end{array}$ & $\begin{array}{l}\text { Óbito } \\
\text { totais }\end{array}$ & $\begin{array}{l}\text { Óbito } \\
<1 \text { ano }\end{array}$ & $\begin{array}{l}\text { Óbito< } \\
\text { 28dias }\end{array}$ & MIP & CMI & CMNN & CMPNN & $\begin{array}{l}\text { Educ. } \\
\text { fund. }\end{array}$ & $\begin{array}{l}\text { Renda } \\
\text { baixa }\end{array}$ & $\begin{array}{c}\text { Centros/ } \\
\text { Postos }\end{array}$ & $\begin{array}{c}\text { L.Obst/ } \\
1.000\end{array}$ & $\begin{array}{l}\text { Saneam. } \\
\text { (2) }\end{array}$ & Favela \\
\hline 60- Sete de Abril & 3 & 367 & 186 & 21 & 8 & 11,3 & 57,2 & 21,8 & 35,4 & 86,9 & 70,1 & 2 & - & 99,2 & 2,0 \\
\hline $\begin{array}{l}\text { 61- Mussurunga/ } \\
\text { S. Crist. }\end{array}$ & 3 & 876 & 181 & 23 & 8 & 12,7 & 26,3 & 9,1 & 17,2 & 87,0 & 61,3 & 3 & - & 98,8 & 23,9 \\
\hline 62- Plataforma & 3 & 1.148 & 320 & 30 & 18 & 9,4 & 26,1 & 15,7 & 10,5 & 87,1 & 69,2 & 2 & - & 99,2 & 12,9 \\
\hline 63- Pirajá & 3 & 431 & 109 & 7 & 4 & 6,4 & 16,2 & 9,3 & 6,9 & 88,9 & 67,9 & - & - & 99,2 & 2,0 \\
\hline 64- Pirajá/P. Seco & 3 & 159 & 16 & 1 & - & 6,3 & 6,3 & - & 5,1 & 88,7 & 60,8 & 2 & - & 98,2 & 14,5 \\
\hline 66- Escada/Periperi & 3 & 944 & 363 & 58 & 22 & 16,0 & 61,4 & 23,3 & 38,1 & 84,6 & 63,9 & 3 & - & 98,6 & 10,4 \\
\hline 68- Valéria & 3 & 212 & 89 & 14 & 5 & 15,7 & 66,0 & 23,6 & 42,4 & 84,7 & 68,4 & 1 & - & 98,3 & 0,6 \\
\hline 69- Águas Claras & 3 & 2.214 & 315 & 38 & 17 & 12,1 & 17,2 & 7,7 & 9,5 & 88,3 & 58,0 & 6 & 0,3 & 99,1 & 12,2 \\
\hline $\begin{array}{l}\text { 7- Nordeste } \\
\text { Amaralina }\end{array}$ & 4 & 867 & 294 & 25 & 11 & 8,5 & 28,8 & 12,7 & 16,1 & 86,1 & 78,8 & 2 & - & 98,9 & 100,0 \\
\hline 30- Pernambués & 4 & 920 & 258 & 35 & 14 & 13,6 & 38,0 & 15,2 & 22,8 & 85,0 & 69,5 & 5 & - & 99,7 & 14,2 \\
\hline 44- Engomadeira & 4 & 1.592 & 351 & 48 & 17 & 13,7 & 30,2 & 10,7 & 19,5 & 85,5 & 65,0 & 1 & - & 99,3 & 18,0 \\
\hline 45- CAB & 4 & 69 & 2 & 1 & - & 50,0 & 14,5 & - & 17,5 & 83,0 & 76,3 & - & - & 100,0 & 27,5 \\
\hline 49- Uruguai & 4 & 960 & 217 & 19 & 15 & 8,8 & 19,8 & 15,6 & 4,2 & 86,1 & 72,6 & 2 & - & 99,0 & 18,6 \\
\hline 52- Mata Escura & 4 & 785 & 160 & 40 & 12 & 25,0 & 51,0 & 15,3 & 35,7 & 83,3 & 62,4 & 3 & - & 98,2 & 19,4 \\
\hline 53- Sussuarana & 4 & 995 & 116 & 18 & 8 & 15,5 & 18,1 & 8,0 & 10,1 & 81,5 & 77,6 & 1 & - & 97,2 & 16,0 \\
\hline 55- Itapuã & 4 & 1.414 & 294 & 35 & 15 & 11,9 & 24,8 & 10,6 & 14,1 & 84,5 & 61,5 & 3 & - & 96,8 & 25,2 \\
\hline 57- Lobato/Pirajá & 4 & 914 & 211 & 21 & 11 & 10,0 & 23,0 & 12,0 & 11,0 & 81,5 & 67,6 & 2 & - & 95,1 & 8,8 \\
\hline 59- Pau da Lima & 4 & 887 & 261 & 36 & 9 & 13,8 & 40,6 & 10,1 & 30,5 & 84,1 & 70,4 & 1 & - & 98,8 & 20,0 \\
\hline 65- Castelo Branco & 4 & 490 & 123 & 16 & 5 & 13,0 & 32,7 & 10,2 & 22,5 & 84,7 & 69,8 & 2 & - & 99,4 & 35,9 \\
\hline 67- São Bartolomeu & 4 & 194 & 11 & 2 & 1 & 18,2 & 10,3 & 5,2 & 5,2 & 78,8 & 71,8 & - & - & 93,1 & 41,9 \\
\hline 70- Est. Cia/Aerop. & 4 & 449 & 21 & 4 & - & 19.0 & 8,9 & - & 10,0 & 73,9 & 75,3 & - & - & 79,0 & 21,9 \\
\hline 71- Coutos & 4 & 1.558 & 172 & 36 & 12 & 20,9 & 23,1 & 7,7 & 15,4 & 77,4 & 74,5 & 1 & - & 99,3 & 7,8 \\
\hline 72- Limite c/ Usiba & 4 & 76 & - & - & - & - & - & - & - & 52,1 & 92,3 & - & - & 95,4 & 89,6 \\
\hline $\begin{array}{l}\text { 73- Paripe/ } \\
\text { Base Naval }\end{array}$ & 4 & 880 & 280 & 45 & 13 & 16,1 & 51,1 & 14,8 & 36,3 & 85,9 & 64,9 & 1 & 0,7 & 96,1 & 20,1 \\
\hline $\begin{array}{l}\text { 74- Ilha Bom } \\
\text { Jesus/Frades }\end{array}$ & 4 & 20 & 4 & - & - & - & - & - & - & 61,2 & 81,9 & - & - & 0,0 & 0,5 \\
\hline 76- Ilha de Maré & 4 & 64 & 5 & 1 & 1 & 20,0 & 15,6 & 15,6 & - & 51,2 & 90,5 & - & - & 0,0 & 0,6 \\
\hline Ignorada & - & - & 79 & 14 & 8 & - & - & - & - & - & - & - & - & - & - \\
\hline Não registrada & - & - & 132 & 73 & 70 & - & - & - & - & - & - & - & - & - & - \\
\hline Total & - & 38.007 & 10.771 & 1.096 & 518 & 10,2 & 28,8 & 13,6 & 15,2 & $x x x$ & $x x x$ & $x x x$ & $x x x$ & $x x x$ & $x x x$ \\
\hline
\end{tabular}

$I C V$ elevado $=1 ; \quad I C V$ intermediário $=2 ; \quad I C V$ baixo $=3 ; \quad I C V$ muito baixo $=4$

1 Refere-se a média dos indicadores das duas ZI;

(2) \% de domicílios ligados à rede de abastecimento de água

Vale salientar que, por se tratar de uma análise de agregados, este estudo está sujeito às limitações de certa forma já apontadas anteriormente, tais como a hetrogeneidade intra-agregados, a mobilidade intergrupos, a dependência da escala utilizada, entre outras, que, de acordo com a epidemiologia, constituem-se em sérios impedimentos para a inferência causal. Todavia, não é demais esclarecer não ter sido este o propósito do presente trabalho, mas, sim, oferecer subsídios para o planejamento e a avaliação da oferta de serviços e para monitorar as condições de saúde infantil.

Assim, em que pese às restrições apresentadas, o estudo da mortalidade infantil considerando-se sua distribuição no espaço geográ- fico entendido como produto de transformações exercidas pelo homem (Santos, 1979) não só permitiu a identificação de áreas da cidade onde residem grupos submetidos a um maior risco, como também contribuiu para evidenciar a manutenção das desigualdades sociais. A constatação de que, apesar da redução observada nos níveis desse tipo de mortalidade, mantêm-se acentuados diferenciais entre os diversos segmentos populacionais é sugestiva de que os processos sociais que comprometem as condições de vida desempenham um papel decisivo na determinação da mortalidade infantil. 


\section{Agradecimentos}

Trabalho realizado com apoio da Organização PanAmericana de Saúde (OPAS)/Conselho Nacional de Desenvolvimento Científico e Tecnológico (CNPq) (processo 521.820/93-6, acordo OPAS/CNPq DRC/ $\mathrm{RPD} / 63 / 5 / 12)$ na primeira etapa e do CNPq na segunda etapa (processo 520997/95-6 NV, com vigência de $1 / 8 / 97$ a $31 / 7 / 99)$.

\section{Referências}

AGEITOS, M. L.; RICO-VELAZCO, J.; OJEDA, N. S. \& YUNES, J., 1991. La Mortalidad de la Niñez en las Américas. Tendencias y Diferenciales. Análisis de Casos. Programa de Salud Maternoinfantil. Washington, DC: Organización Panamericana de la Salud/Organización Mundial de la Salud.

ANUÁRIO DELTA UNIVERSAL, 1992. Economia. Rio de Janeiro: Edições Delta.

BARROS, F. C.; VICTORA, C. G.; CESAR, J. A. \& HORTA, B. L., 1993. Diagnóstico de Saúde das Crianças e Mulheres da Bahia. Salvador: Sistema Único de Saúde, Secretaria Estadual de Saúde da Bahia, Governo da Bahia/Fundo das Nações Unidas para a Infância/Universidade Federal de Pelotas/ Editora Gráfica da Bahia.

BEHM, H., 1980. Determinantes económicas y sociales de la mortalidad en América Latina. Revista Cubana de Salud, 6:1-30.

BREILH, J., 1990. Pobreza urbana y salud: Una mirada desde la epidemiología crítica. In: 10o Congresso Brasileiro de Epidemiologia, Anais, pp. 281-302. Campinas: ABRASCO.

CASTELLANOS, P. L., 1990. Avances metodológicos en epidemiología. In: 10o Congresso Brasileiro de Epidemiologia, Anais, pp. 201-216. Campinas: ABRASCO.

GONÇALO-PEREZ, G. \& HERRERA-LEON, L., 1990. Desarrollo social y mortalidad infantil, 1977-1986, Cuba. Un análisis regional. Revista de Saúde Pública, 24:186-195.

GRANT, J., 1992. Situação Mundial da Infância, 1993. Brasília: Fundo das Nações Unidas para a Infância.

GRASSI, P. R. \& LAURENTI, R., 1998. Implicações da introdução da 10a Revisão da Classificação Internacional de Doenças em análise de tendência da mortalidade por causas. Informe Epidemiológico do SUS, 7:43-47.

GUIMARÃES, J. J. L. \& FISCHMANN, A., 1986. Desigualdades na mortalidade infantil entre favelados e não favelados no Município de Porto Alegre, Rio Grande do Sul, em 1980. Boletín de la Oficina Sanitaria Panamericana, 10:19-38.

IBGE (Fundação Instituto Brasileiro de Geografia e Estatística), 1991. Censo Demográfico. Rio de Janeiro: IBGE.
LARDELLI, P.; BLANCO, J. I.; DELGADO-RODRIGUEZ, M.; BUENO, A.; LUNA, J. D. \& GALVEZ, R., 1993. Influence of socioeconomic and health care development on infant and perinatal mortality in Spain 1975-1986. Journal of Epidemiology and Community Health, 47:260-264.

LIMA, B. G. \& COSTA, M. C. N., 1999. Mortalidade infantil e seus componentes em Salvador-BA, 19801991. Informe Epidemiológico do SUS, 7:35-41.

MONTEIRO, C. A., 1982. Contribuição para o estudo do significado do coeficiente da mortalidade infantil no Município de São Paulo, SP (Brasil), nas três últimas décadas (1950-1979). Revista de Saúde Púbica, 16:7-18.

MONTEIRO, C. A.; BENÍCIO, M. H. A. \& BALDIJÃO, M F. A., 1980. Mortalidade no primeiro ano de vida e a distribuição de renda e de recursos públicos de saúde, São Paulo (Brasil). Revista de Saúde Pública,14:515-539.

MS (Ministério da Saúde), 1998. Sistema de Informações sobre Mortalidade 1979-1997. CD-ROM. Brasília: Departamento de Informática do SUS.

MURRAY, C. J. L., 1988. The infant mortality rate, life expectancy at birth and a linear index of mortality as measures of general health status. International Journal of Epidemiology, 17:122-128.

OPS (Organización Panamericana de la Salud), 1992. Metodología para el estudio de desigualdades en la situación de salud. Boletín Epidemiológico, 12:7-10.

PAIM, J. S., 1997. Abordagens teórico-conceituais em estudos de condições de vida e saúde: Algumas notas para reflexão e ação. In: Condições de Vida e Situação de Saúde (R. Barata, org.), pp. 7-30, Rio de Janeiro: ABRASCO.

PAIM, J. S. \& COSTA, M. C. N., 1993. Decline and unevenness of infant mortality in Salvador, Brazil, 1980-1988. Bulletin of the Pan-American Health Organization, 27:1-14.

PAIM, J. S.; COSTA, M. C. N.; CABRAL, V.; MOTA, I. A. \& BATISTA-NEVES, R. B., 1987. Spatial distribution of proportional infant mortality and certain socioeconomic variables in Salvador, Bahia, Brazil. Bulletin of the Pan-American Health Organization, 21:225-239. 
SANTOS, M., 1979. Espaço e Sociedade: Ensaios. Petrópolis: Editora Vozes.

SANTOS, M., 1980. Por uma Geografia Nova. São Paulo: Editora Hucitec.

SEI (Superintendência de Estatística e Informações), 1999. Anuário Estatístico. Salvador: SEI.

SESAB (Secretaria de Saúde do Estado da Bahia), 1991. Anuário Estatístico - Informações de Saúde, BA, 1976-1990. Salvador: Centro de Informações de Saúde, Secretaria de Saúde do Estado da Bahia.

SESAB (Secretaria de Saúde do Estado da Bahia), 1995. Anuário Estatístico - Informações de Saúde, BA. Salvador: Centro de Informações de Saúde, Secretaria de Saúde do Estado da Bahia.

SES-RS (Secretaria de Estado da Saúde do Rio Grande do Sul), 1993. Estatística de Saúde. Mortalidade, 1991. v.17, Porto Alegre: Núcleo de Informática, Divisão de Informação e Saúde, Departamento de Planejamento, Secretaria de Estado da Saúde do Rio Grande do Sul.

SILVA, A. L. C. \& DURAN, M. S., 1990. Mortalidad infantil y condiciones higiénico-sociales en las Américas. Un estudio de correlación. Revista de Saúde Pública, 24:473-480.

SPSS INCORPORATION, 1998. SPSS for Windows. Statistical Package for the Social Sciences. Release 9.0. Chicago: SPSS Incorporation.

UNICEF (Fundo das Nações Unidas para a Infância), 1994. Situação Mundial da Infância. Brasília: UNICEF.
VICTORA, C. G.; GRASSI, P. R. \& SCHMIDT, A. M., 1994. Situação de saúde da criança em área da Região Sul do Brasil, 1980-1992: Tendências temporais e distribuição espacial. Revista de Saúde Pública, 28:423-432.

VIEIRA-DA-SILVA, L. M.; FORMIGLI, V. L. A; CERQUEIRA, M. P.; KRUCHEVSKY, L.; TEIXEIRA, M. M. A.; BARBOSA, A. S. M.; CONCEIÇÃO, P. S. A.; KHOURI, M. A. \& NASCIMENTO, C. L., 1995. O processo de distritalização - Avaliação do caso de Pau da Lima, Salvador, Bahia, Brasil. Cadernos de Saúde Pública, 11:72-84.

WHO (World Health Organization), 1991. Surveillance of living conditions and the health situation. Epidemiological Bulletin, 12:7-10.

WHO (World Health Organization), 1993. World Health Statistics Annual, 1992. Geneva: WHO.

WHO (World Health Organization), 1994. Epi Info Version 6.02. A Word Processing, Database, and Statistics System for Epidemiology on Microcomputers. Geneva: WHO.

YUNES, J., 1983. Características sócio-econômicas da mortalidade infantil em São Paulo. Pediatria, 5: 162-168.

ZURRIAGA-LLORENS, O.; ALFONSO-SANCHEZ, J. L.; SANCHIS-NOGUERA, B.; PRADO-DEL-BAÑO, M. J. \& CORTINA-GREUS, P., 1990. Algunos factores determinantes de la mortalidad infantil en España. Salud Pública, 32:665-672. 